\title{
Interprofessional collaboration within teams comprised of health and other professionals: a systematic review of measurement tools and their psychometric properties
}

\author{
Julia Jacob \\ University of South Australia, jjacob@outlook.com.au \\ Kobie Boshoff \\ University of South Australia \\ Rebecca Stanley \\ University of Wollongong \\ Hugh Stewart \\ University of South Australia \\ Louise Wiles \\ University of South Australia \\ Follow this and additional works at: https://nsuworks.nova.edu/ijahsp \\ Part of the Medicine and Health Sciences Commons
}

This Systematic Review has supplementary content. View the full record on NSUWorks here: https://nsuworks.nova.edu/ijahsp/vol15/iss2/8

\section{Recommended Citation}

Jacob J, Boshoff K, Stanley R, Stewart H, Wiles L. Interprofessional collaboration within teams comprised of health and other professionals: a systematic review of measurement tools and their psychometric properties. The Internet Journal of Allied Health Sciences and Practice. 2017 Jan 01;15(2), Article 8.

This Systematic Review is brought to you for free and open access by the College of Health Care Sciences at NSUWorks. It has been accepted for inclusion in Internet Journal of Allied Health Sciences and Practice by an authorized editor of NSUWorks. For more information, please contact nsuworks@nova.edu. 


\title{
Interprofessional collaboration within teams comprised of health and other professionals: a systematic review of measurement tools and their psychometric properties
}

\begin{abstract}
Background and Purpose: Measuring collaboration within interprofessional teams allows professionals to evaluate their practice, set benchmarks and improve outcomes. In the context of healthcare, most research has focused on teams comprised solely of health professionals, with limited attention given to collaboration between health and other professionals. Given the escalating complexities of healthcare, and the growing need for interprofessional collaborative practice involving team members external to health care, this represents a considerable gap in the literature. Therefore, the purpose of this review was to identify tools that measure collaboration within interprofessional teams comprised of members from health and other disciplines, and evaluate their psychometric properties. This review focused on the area of children's services, to assist professionals working in this area with their collaborative practice. Methods: A systematic search including nineteen electronic databases was conducted. Eleven articles (describing ten tools) were identified for inclusion and were critically appraised. Results: Overall, it was found that few psychometrically sound tools exist for more diverse professional groups working together. The PINCOMQ was found to be the most appropriate tool for the context of children's services, and with the highest critical appraisal score, as reported. Conclusions: Recommendations are made for further development of existing tools before practical implementation. Further research could develop new and innovative tools to accommodate the evolving composition of future interprofessional teams.
\end{abstract}

\footnotetext{
Author Bio(s)

Julia Jacob, BAppSc (OT) Hons is a practicing paediatric occupational therapist, who completed her honours thesis at the University of South Australia, South Australia.

Dr Kobie Boshoff, PhD, is a Senior Lecturer in the Occupational Therapy Program at the University of South Australia and a researcher within the International Centre for Allied Health Evidence.

Mr Hugh Stewart, M App Sc (OT), is the Program Director for the Occupational Therapy Program at the University of South Australia and a researcher within the International Centre for Allied Health Evidence.

Dr Rebecca Stanley, PhD, is an Associate Research Fellow, at the Early Start Research Institute (ESRI) at the University of Wollongong.

Dr Louise Wiles, PhD, is the Project Manager for the Patient Safety and Healthcare Human Factors Centre for Population Health Research at the University of South Australia.
} 


\title{
IUAHSP \\ The Internet Joưnal of Allied Health Sciences and Practice
}

Dedicated to allied health professional practice and education

Vol. 15 No. 2 ISSN 1540-580X

\section{Interprofessional Collaboration within Teams Comprised Of Health and Other Professionals: A Systematic Review of Measurement Tools and Their Psychometric Properties}

\author{
Julia Jacob, BAppSc ${ }^{1}$ \\ Kobie Boshoff, PhD1 \\ Rebecca StanleyPhD2 \\ Hugh Stewart ${ }^{1}$ \\ Louise Wiles $\mathrm{PhD}^{1}$
}

1. University of South Australia

2. University of Wollongong

Australia

\begin{abstract}
Background and Purpose: Measuring collaboration within interprofessional teams allows professionals to evaluate their practice, set benchmarks and improve outcomes. In the context of healthcare, most research has focused on teams comprised solely of health professionals, with limited attention given to collaboration between health and other professionals. Given the escalating complexities of healthcare, and the growing need for interprofessional collaborative practice involving team members external to health care, this represents a considerable gap in the literature. Therefore, the purpose of this review was to identify tools that measure collaboration within interprofessional teams comprised of members from health and other disciplines, and evaluate their psychometric properties. This review focused on the area of children's services, to assist professionals working in this area with their collaborative practice. Methods: A systematic search including nineteen electronic databases was conducted. Eleven articles (describing ten tools) were identified for inclusion and were critically appraised. Results: Overall, it was found that few psychometrically sound tools exist for more diverse professional groups working together. The PINCOM-Q was found to be the most appropriate tool for the context of children's services, and with the highest critical appraisal score, as reported. Conclusions: Recommendations are made for further development of existing tools before practical implementation. Further research could develop new and innovative tools to accommodate the evolving composition of future interprofessional teams.
\end{abstract}

\section{Introduction}

Interprofessional collaboration in health care is defined as "the process in which different professional groups work together to positively impact health care", and also adding to this definition, "...each (profession) making unique contributions to common goals."1,2 There is a prevalent view that interprofessional collaboration leads to enhancements in health care such as gains in quality of care and patient safety and has positive associations with the satisfaction of patients and staff, as well as improvements in clinical outcomes and greater patient outcomes.2-5 Whilst these definitions and benefits derive from health care, health professionals often need to collaborate with other professionals outside health to deliver services to client groups. A cohesive and holistic approach is often required in the area of children's services, focussing on the family and community, resulting in the need to tap into a variety of interdisciplinary expertise, from professional areas such as education, health and welfare. 6 Since children's services has been noted by a large number of authors as complex and challenging, our focus has been on the area of children's services in order to assist professionals working in this area.7-9 An example of such a setting is the South Australian Children's Centres. Children's Centres is a state government initiative that provides a variety of services (including education and health), to children and families in a single familiar setting within the community. Thus, Children's Centres contain a unique team environment where professionals from different backgrounds/disciplines work together, requiring interprofessional

(c) The Internet Journal of Allied Health Sciences and Practice, 2017 
collaboration. Team members at these sites may include occupational therapists, speech language pathologists, educators, and welfare staff as well as child care staff members.

In health literature, the benefits, need for, and importance of effective collaboration has been documented. Environments with poor collaboration have shown to have decreased levels of patient care and provision of health services.1,10 Based on this evidence, it is important for teams to continually strive towards optimal collaboration. In order to do this, professionals within teams need to be able to reflect on and understand their level of collaboration, and its impact on practice.11 Literature should be used to guide practitioners in developing their collaborative practice. However, most literature is related to health settings, with less known about interprofessional collaboration between diverse professional groups, including health professionals collaborating with those external to health.

A self-evaluation tool to measure or describe a team's interprofessional collaboration would be beneficial for their reflection on and development of their collaborative practice, a strategy supported by authors in this field. 6 Prior reviews on collaboration tools have either focussed only on healthcare settings, interprofessional education, or a combination of the two.12-16 Known tools originate mostly from a health care perspective and/or include only health care professionals as team members; examples include the Communication and Teamwork Skills Assessment (CATS), the Observational Teamwork Assessment of Surgery (OTAS) - a speciality specific tool, and the Collaborative Practice Assessment Tool (CPAT) developed by the Office of Interprofessional Education at the Queens University.17-19

In order to assist professionals working in interprofessional teams consisting of a variety of diverse professional backgrounds to critically reflect on their collaboration and consequently work on their team development, it was necessary to conduct a review to find a relevant interprofessional collaboration measurement tool. Given the increased need for interprofessional collaboration due to escalating complexities of current day healthcare - such as staff shortages and the growing demand for services, as well as in addressing the multi-faceted complexities of health problems, this review is intended to address a significant gap in the literature.20,21

\section{METHOD:}

\section{Data Sources and Search Strategy}

The protocol for this review was developed, conducted, and reported according to the Preferred Reporting ltems for Systematic Reviews and Meta-Analyses (PRISMA) statement.21The search terms were identified by referring to collaboration literature and were based on personal experience in the field. A scoping search was conducted after which all authors agreed upon the final terms, and an expert librarian vetted the terms to ensure the terms were correct and exhaustive. Because of the inconsistencies of terms within the literature, the search was kept broad and then narrowed down with selection criteria (Appendix 1). Database platforms searched included Ovid, EBSCO, Web of knowledge, Cochrane Library, Informit, and Scopus. Under the guidance of our consultant academic librarian, any database deemed likely to index potentially relevant articles was included to help facilitate adequate capture of the published literature. Nineteen electronic databases were searched (Appendix 2) using key words, and where available, Medical Subject Headings (MeSH). The search strategy, first developed for Medline, was adapted for all other database platforms in order to accommodate their unique search criteria. Searches were conducted between February 23 and March 20, 2014.

\section{Selection Criteria and Process}

Articles were included if they were primary studies which reported on the development processes and psychometric testing of measurement tools for interprofessional collaboration in teams comprising health and other professionals. Health professionals included any profession within the medical or allied health field (for example, nurse, occupational therapist, and speech pathologist) and other professions included anyone from outside this field (for example teacher, child care worker). Tools were included if they measured collaboration via self-evaluation of the team's collaboration with each other, therefore excluding studies which focus on professionals' attitudes towards, and views on, theoretical collaboration. Additional inclusion criteria included peer-reviewed articles, publication in English, and publication within the last ten years (2004 to 2014 inclusive) to ensure our review was based on current literature. Reference lists were reviewed to identify any further relevant studies.

PRISMA guidelines were followed within the review process (refer to the flow chart in Figure 1). A total of 16,366 articles were identified (Figure 1). Duplicates were removed electronically and double checked manually, and non-peer reviewed articles were excluded. Following this, articles were examined against eligibility criteria on title level by two of the authors independently, then also on abstract level with 1,131 articles. Any discrepancies were resolved by sourcing the article for full text review and discussion. If titles or abstracts provided insufficient detail to determine suitability, they were retained. A total of 80 articles were included for review of the full-text. Four authors were independently involved in the full text review process, ensuring each article

(c) The Internet Journal of Allied Health Sciences and Practice, 2017 
was screened by two authors. Articles were excluded if they did not meet the selection criteria as agreed by reviewers. Any debate about inclusion was resolved through discussion and consensus.

\section{Figure 1 - Adapted PRISMA Flow Diagram}

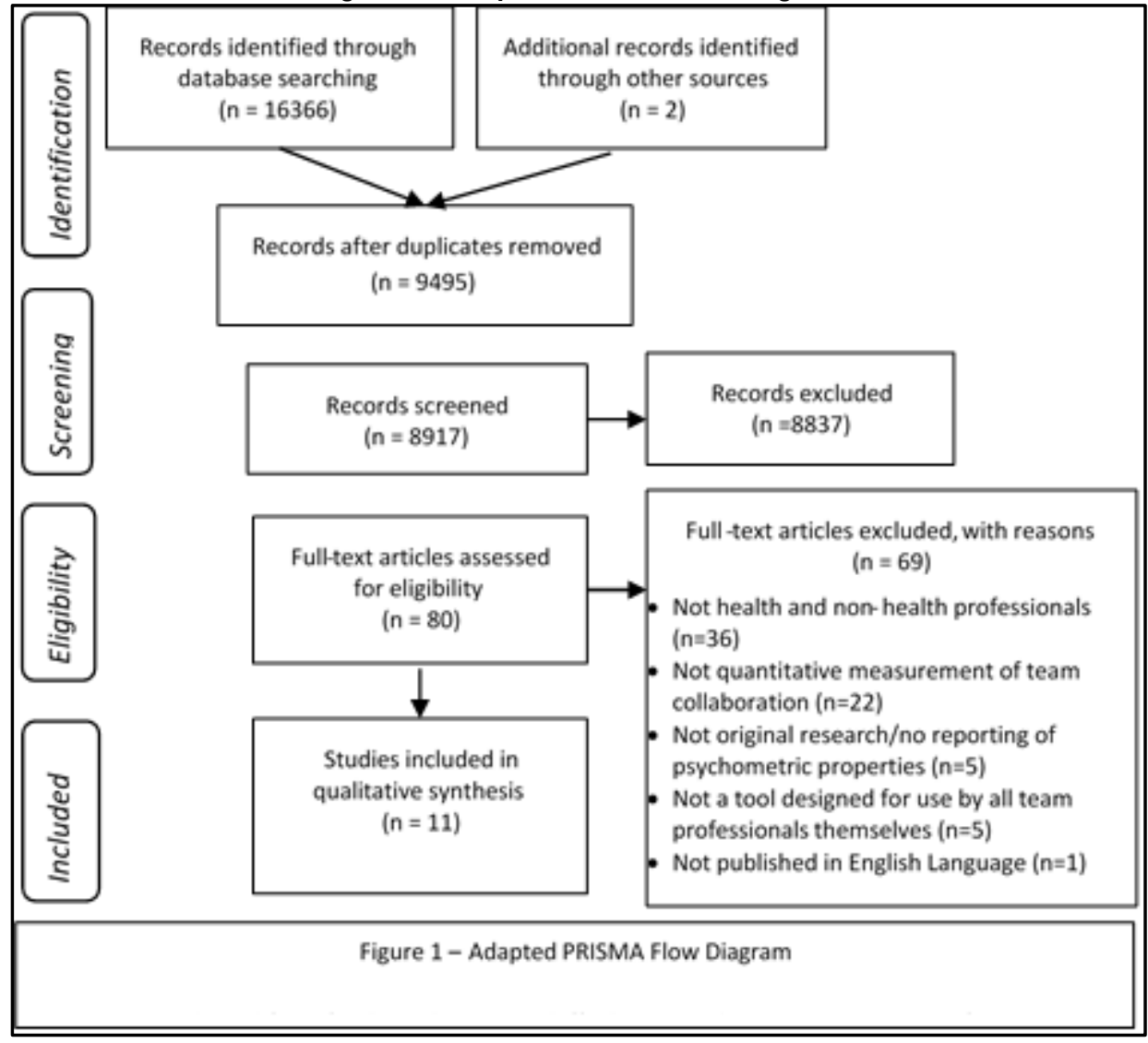

\section{Data Extraction:}

Data were extracted from the selected articles by the first author and then reviewed by one of the other authors. Information extracted was determined by common themes or areas which the articles reported on as well as the information needed for critical appraisal such as psychometric properties.

\section{Critical Appraisal}

There are limited tools available for critical appraisal of questionnaire development research. Current tools do not include items that considered all aspects of psychometric testing deemed relevant by the authors, such as relevance to the setting, piloting samples, and layout of the questionnaire. Therefore, a purpose-designed critical appraisal tool was developed for use in the current study using firstly the McMaster Critical Review Form (quantitative) - a form used extensively in reviews and which has undergone testing by the McMaster University, and secondly, guiding literature regarding questionnaire design, as well as the use of an example of a questionnaire development study (refer Appendix 3 for the critical appraisal tool).23-25 The critical appraisal tool contained six sub categories including purpose, validity, reliability, external validity, layout, and piloting. Under each category there was a series of criteria accompanied by weighted scoring options. Larger critical appraisal scores were considered to represent greater methodological rigor (i.e. reliability and validity) and relevance for applied settings.

In order to capture items relevant to teamwork comprising health and other disciplines in children's services, the external validity section of the critical appraisal tool was developed with reference to key publications referring to health and non-health professionals in these services.11,23-25 These items were that the setting was not purely health, that the sample had adequate representation of health and other professionals, that the sample worked in a child-based setting, and that team members were employed by different agencies. Four of the authors were involved in developing the critical appraisal tool and thus had a detailed understanding of the scoring process. Two authors independently appraised all included articles. Each article was assigned a score out of 70 points by each author, with averages calculated to enhance methodological rigor. A variance of $15 \%$ or more 
was deemed to warrant further discussion and re-evaluation by the reviewers, disagreements were resolved by discussion and consensus to ensure reviews scores were within less than $15 \%$ variance.

\section{RESULTS:}

\section{Characteristics of the Studies}

Ten measurement tools were described within 11 peer reviewed articles. Two articles discussed the same measurement tool, the PINCOM-Q, using different methods and samples.26-27 The majority of included articles ( $n=6,54 \%$ ) were published within the last five years (i.e. 2009 - 2014) (Table 1).19,27-31 The main areas of practice in which tools were tested varied and included health promotion, geriatric care, child and youth mental health, assistive communication, mental health, and hospital based teams.19,26-34,36

Three of the eleven articles did not detail the composition of interdisciplinary teams. Where reported, health professionals within teams commonly included nurses, social workers, doctor/physicians, psychologists, occupational therapists, speech therapists, physical therapists, dieticians, physiotherapists, pharmacists, and allied health aides (Table 1).28-29,36 Along the spectrum of other professional team members described were those working in the following roles: spiritual provider/chaplain, education/teachers, administration staff, volunteers, driver/porter, managers, child welfare staff, computer technicians, art therapists, social services staff, cleaners, and activity coordinator. The sub-scales of tools included a range of different constructs, with the most common being leadership $(n=4)$, communication $(n=3)$, goals $(n=3)$, role independence $(n=3)$, decision making $(n=3)$, team structures $(n=3)$, conflict $(n=3)$, motivation $(n=2)$, coordination $(n=2)$, client involvement $(n=2)$, organisational aspects $(n=2)$ and reflection $(n=2)$ (Table 2$)$.

There were a range of psychometric assessment approaches used across the tools (Table 2) and included face, content, and construct validity, as well as internal consistency. The majority of studies conducted factor analysis on the tools, using either principal component analysis (PCA, $n=5$ ) or exploratory factor analysis (EFA, $n=3$ ), few in addition employed confirmatory factor analysis (CFA, $n=2) \cdot 19,26,28-31,34-35$ 
Table 1 - Characteristics of the Included Studies

\begin{tabular}{|c|c|c|c|c|c|c|c|}
\hline Tool & Author & Location & Setting & Participants & $\begin{array}{l}\text { Sample } \\
\text { Size }\end{array}$ & $\begin{array}{c}\text { Theoretical } \\
\text { model }\end{array}$ & $\begin{array}{l}\text { Internal } \\
\text { structure }\end{array}$ \\
\hline $\begin{array}{l}\text { Perception of } \\
\text { Interprofessional } \\
\text { Collaboration } \\
\text { Model } \\
\text { Questionnaire } \\
(\text { PINCOM-Q })^{27}\end{array}$ & Ødegård, A & $\begin{array}{l}\text { Western } \\
\text { Norway }\end{array}$ & $\begin{array}{l}\text { Professionals } \\
\text { engaged in } \\
\text { interprofessional } \\
\text { collaboration in } \\
\text { relation to } \\
\text { children with } \\
\text { mental health } \\
\text { problems }\end{array}$ & $\begin{array}{l}\text { Primary care, } \\
\text { specialist services } \\
\text { or schools. } \\
\text { Including; teachers, } \\
\text { special educators, } \\
\text { psychologists, } \\
\text { social workers, } \\
\text { primary nurses, } \\
\text { child welfare } \\
\text { workers, medical } \\
\text { doctors. }\end{array}$ & 134 & $\begin{array}{l}\text { PINCOM } \\
\text { theoretical } \\
\text { model }^{26} \\
\text { (Ødegård, } \\
\text { 2005) }\end{array}$ & $\begin{array}{l}48 \\
\text { questions } \\
7 \text { point } \\
\text { Likert scale }\end{array}$ \\
\hline $\begin{array}{l}\text { Teamwork in } \\
\text { Assertive } \\
\text { Community } \\
\text { Treatment } \\
\text { (TACT) Scale } 32\end{array}$ & $\begin{array}{l}\text { Wholey, D } \\
\text { Zhu, X } \\
\text { Knoke, D } \\
\text { Shah, P } \\
\text { Zellmer- } \\
\text { Bruhn, M } \\
\text { Witheridge, T }\end{array}$ & $\begin{array}{l}\text { Minnesota } \\
\text { USA }\end{array}$ & $\begin{array}{l}\text { Mental health } \\
\text { rehabilitation }\end{array}$ & $\begin{array}{l}\text { Assertive } \\
\text { Community } \\
\text { Treatment teams. } \\
\text { Multidisciplinary } \\
\text { but no details of } \\
\text { professions. May } \\
\text { include } \\
\text { professionals from; } \\
\text { mental health, } \\
\text { substance abuse, } \\
\text { supported } \\
\text { employment, social } \\
\text { services and } \\
\text { nursing. }\end{array}$ & $\begin{array}{l}\text { Wave } 1 \\
287 \\
\text { Wave } 2 \\
268 \\
\text { Wave } 3 \\
275\end{array}$ & $\begin{array}{l}\text { Authors } \\
\text { developed } \\
\text { conceptual } \\
\text { model of } \\
\text { team } \\
\text { processes } \\
\text { moderating } \\
\text { and } \\
\text { mediating the } \\
\text { relationship } \\
\text { between ACT } \\
\text { design fidelity } \\
\text { and } \\
\text { performance. }\end{array}$ & $\begin{array}{l}43 \\
\text { questions } \\
\text { (Variety of } \\
\text { question } \\
\text { formats } \\
\text { included; } \\
\text { Likert, open } \\
\text { ended, } \\
\text { interval } \\
\text { scale.) }\end{array}$ \\
\hline $\begin{array}{l}\text { Index of } \\
\text { Interprofessional } \\
\text { Team } \\
\text { Collaboration for } \\
\text { Expanded } \\
\text { School Mental } \\
\text { Health (IITC- } \\
\text { ESMH) } \\
{ }^{31}\end{array}$ & $\begin{array}{l}\text { Mellin, E A } \\
\text { Bronstein, L } \\
\text { Anderson- } \\
\text { Butcher, D } \\
\text { Amorose, A } \\
\text { J } \\
\text { Ball, A } \\
\text { Green, J }\end{array}$ & USA & $\begin{array}{l}\text { Expanded School } \\
\text { Mental Health. }\end{array}$ & $\begin{array}{l}\text { Social work, } \\
\text { counselling, } \\
\text { nursing, } \\
\text { psychology, } \\
\text { education. Either } \\
\text { school employed or } \\
\text { community based. }\end{array}$ & 436 & $\begin{array}{l}\text { Bronstein's } \\
\text { Model of } \\
\text { Interdisciplin } \\
\text { ary } \\
\text { Collaboration } \\
\text { (Bronstein, } \\
2003 \text { ) }\end{array}$ & $\begin{array}{l}26 \\
\text { questions. } \\
5 \text { point } \\
\text { Likert scale }\end{array}$ \\
\hline $\begin{array}{l}\text { Team Decision } \\
\text { Making } \\
\text { Questionnaire } \\
(\text { TDMQ) }\end{array}$ & $\begin{array}{l}\text { Batorowicz, } \\
\text { B } \\
\text { Shepherd, T } \\
\text { A }\end{array}$ & $\begin{array}{l}\text { Ontario, } \\
\text { Canada }\end{array}$ & $\begin{array}{l}\text { Argumentative } \\
\text { and Alternative } \\
\text { Communication } \\
\text { (AAC) centres. }\end{array}$ & $\begin{array}{l}\text { Speech language } \\
\text { pathologists, } \\
\text { occupational } \\
\text { therapists, } \\
\text { communicative } \\
\text { disorders } \\
\text { assistants, } \\
\text { educators, } \\
\text { computer } \\
\text { technicians and } \\
\text { clinic managers. }\end{array}$ & 102 & & $\begin{array}{l}19 \\
\text { questions } \\
7 \text { point } \\
\text { Likert scale }\end{array}$ \\
\hline $\begin{array}{l}\text { PINCOM-Q } \\
\text { (revised) }^{28}\end{array}$ & $\begin{array}{l}\text { Rousseau, C } \\
\text { Lauurin- } \\
\text { Lamothe, A } \\
\text { Nadeau, L } \\
\text { Deshaies, S } \\
\text { Measham, T }\end{array}$ & $\begin{array}{l}\text { Quebec, } \\
\text { Canada }\end{array}$ & $\begin{array}{l}\text { Health and Social } \\
\text { Service Centre } \\
\text { (CSSS) child and } \\
\text { youth teams }\end{array}$ & $\begin{array}{l}\text { Social workers, } \\
\text { psycho educators, } \\
\text { psychologists, art } \\
\text { therapists, nurses } \\
\text { and consulting } \\
\text { child psychiatrists. }\end{array}$ & 96 & $\begin{array}{l}\text { PINCOM } \\
\text { theoretical } \\
\text { model } \\
\text { (Ødegård, } \\
2005 \text { ) }\end{array}$ & $\begin{array}{l}48 \text { items } \\
7 \text { point } \\
\text { Likert } \\
\text { scale. }\end{array}$ \\
\hline
\end{tabular}




\begin{tabular}{|c|c|c|c|c|c|c|c|}
\hline $\begin{array}{l}\text { Professional } \\
\text { Practice } \\
\text { Environment } \\
\text { (PPE) revised } \\
\text { Scale }^{36}\end{array}$ & $\begin{array}{l}\text { Erickson, JI } \\
\text { Duffy, M E } \\
\text { Gibbons, M } \\
\text { P } \\
\text { Fitzmaurice, } \\
\text { J } \\
\text { Ditomassi, M } \\
\text { Jones, D }\end{array}$ & $\begin{array}{l}\text { Boston, } \\
\text { USA. }\end{array}$ & Hospital & $\begin{array}{l}\text { Nursing, } \\
\text { occupational } \\
\text { therapy, physical } \\
\text { therapy, respiratory } \\
\text { therapy, social } \\
\text { services, speech } \\
\text { pathology and } \\
\text { chaplaincy. }\end{array}$ & 849 & $\begin{array}{l}\text { Professional } \\
\text { Practice } \\
\text { Model }\end{array}$ & $\begin{array}{l}38 \text { items } \\
4 \text { point } \\
\text { Likert scale }\end{array}$ \\
\hline $\begin{array}{l}\text { Collaborative } \\
\text { Practice } \\
\text { Assessment Tool } \\
(\text { CPAT })^{14}\end{array}$ & $\begin{array}{l}\text { Schroder, C } \\
\text { Medves, J } \\
\text { Paterson, M } \\
\text { Byrnes, V } \\
\text { Chapman, C } \\
\text { O'Riordan, A } \\
\text { Pichora, D } \\
\text { Kelly, C }\end{array}$ & $\begin{array}{l}\text { Ontario, } \\
\text { Canada }\end{array}$ & $\begin{array}{l}\text { Pilot } 1 \text { - palliative } \\
\text { care team, } \\
\text { geriatric } \\
\text { assessment } \\
\text { team, and two } \\
\text { family practice } \\
\text { teams. } \\
\text { Pilot } 2 \text { - six } \\
\text { participating } \\
\text { healthcare units } \\
\text { from different } \\
\text { clinical practice } \\
\text { settings. }\end{array}$ & $\begin{array}{l}\text { Pilot 1- } \\
\text { administration, } \\
\text { medicine, nursing, } \\
\text { nutrition services, } \\
\text { occupational } \\
\text { therapy, physical } \\
\text { therapy, social } \\
\text { work, spiritual care } \\
\text { and volunteer } \\
\text { services. } \\
\text { Pilot 2-(same as } \\
\text { above plus) } \\
\text { nurses, } \\
\text { physiotherapists, } \\
\text { care staff, } \\
\text { dieticians, } \\
\text { cleaners, } \\
\text { pharmacists, } \\
\text { assistants, } \\
\text { occupational } \\
\text { therapy assistants, } \\
\text { psychologists, } \\
\text { recreation } \\
\text { therapists, } \\
\text { manager, porter, } \\
\text { vocational rehab } \\
\text { worker, ward aide. }\end{array}$ & $\begin{array}{l}\text { Pilot } 1 \\
42 \\
\\
\text { Pilot } 2 \\
111\end{array}$ & & $\begin{array}{l}56 \text { items. } \\
7 \text { point } \\
\text { Likert } \\
\text { scale. As } \\
\text { well as } \\
\text { three open } \\
\text { ended } \\
\text { questions. }\end{array}$ \\
\hline $\begin{array}{l}\text { Modified Index } \\
\text { for } \\
\text { Interdisciplinary } \\
\text { Collaboration } \\
\text { (MIIC) }{ }^{33}\end{array}$ & $\begin{array}{l}\text { Oliver, D P } \\
\text { Wittenberg- } \\
\text { Lyles, E M } \\
\text { Day, M }\end{array}$ & USA & $\begin{array}{l}\text { Hospice } \\
\text { Programs }\end{array}$ & $\begin{array}{l}\text { Nurse, social } \\
\text { worker, chaplain, } \\
\text { aide, doctor of } \\
\text { medicine, } \\
\text { bereavement, } \\
\text { dietary, volunteer, } \\
\text { therapist, team } \\
\text { leader and } \\
\text { secretary. }\end{array}$ & 95 & $\begin{array}{l}\text { Bronstein's } \\
\text { Model of } \\
\text { Interdisciplin } \\
\text { ary } \\
\text { Collaboration } \\
\text { (Bronstein, } \\
\text { 2003) }\end{array}$ & $\begin{array}{l}42 \\
\text { questions }\end{array}$ \\
\hline $\begin{array}{l}\text { Adapted ICU } \\
\text { nurse-physician } \\
\text { questionnaire } \\
\text { (For PACE } \\
\text { teams) }\end{array}$ & $\begin{array}{l}\text { Temkin- } \\
\text { Greener, H } \\
\text { Gross, D } \\
\text { Kunitz, S J } \\
\text { Mukamel, D }\end{array}$ & USA & $\begin{array}{l}\text { Teams within the } \\
\text { Program of All- } \\
\text { Inclusive Care for } \\
\text { the Elderly } \\
\text { (PACE) in long } \\
\text { term care } \\
\text { settings. }\end{array}$ & $\begin{array}{l}\text { Aides, social work, } \\
\text { nurse, physician, } \\
\text { nurse practitioner, } \\
\text { therapist, activity } \\
\text { coordinator, driver } \\
\text { and other. }\end{array}$ & 1220 & $\begin{array}{l}\text { Conceptual } \\
\text { model of } \\
\text { managerial } \\
\text { and team } \\
\text { process } \\
\text { factors } \\
\text { affecting } \\
\text { performance. } \\
\text { (Shortell et } \\
\text { al. 1991) } \\
\end{array}$ & $\begin{array}{l}59 \text { items } \\
5 \text { point } \\
\text { Likert scale }\end{array}$ \\
\hline $\begin{array}{l}\text { Jones Synergy } \\
\text { Scale }^{29}\end{array}$ & $\begin{array}{l}\text { Jones, J } \\
\text { Barry, M M }\end{array}$ & $\begin{array}{l}\text { Republic } \\
\text { of Ireland }\end{array}$ & $\begin{array}{l}\text { Health promotion } \\
\text { partnerships }\end{array}$ & $\begin{array}{l}\text { Representatives } \\
\text { from health, } \\
\text { community, } \\
\text { education, arts, } \\
\text { sports and youth } \\
\text { sectors. }\end{array}$ & 312 & & $\begin{array}{l}8 \text { questions } \\
5 \text { point } \\
\text { Likert scale }\end{array}$ \\
\hline
\end{tabular}




\begin{tabular}{|c|c|c|c|c|c|c|}
\hline $\begin{array}{l}\text { Jones Trust } \\
\text { Scale }^{30}\end{array}$ & $\begin{array}{l}\text { Jones, J } \\
\text { Barry, M M }\end{array}$ & $\begin{array}{l}\text { Republic } \\
\text { of Ireland }\end{array}$ & $\begin{array}{l}\text { Health promotion } \\
\text { partnerships }\end{array}$ & $\begin{array}{l}\text { Participants were } \\
\text { from: hospitals, } \\
\text { community health } \\
\text { services, health } \\
\text { service managers, } \\
\text { education, youth } \\
\text { sector, sports, arts } \\
\text { and voluntary } \\
\text { groups. }\end{array}$ & 270 & $\begin{array}{l}14 \\
\text { questions } \\
5 \text { point } \\
\text { Likert scale }\end{array}$ \\
\hline
\end{tabular}

Table 2 - Psychometric Properties of the Questionnaires

\begin{tabular}{|c|c|c|c|c|c|}
\hline \multirow[b]{2}{*}{ Tool } & \multirow{2}{*}{$\begin{array}{l}\text { Sub Scales } \\
\text { (Elements of } \\
\text { collaboration) }\end{array}$} & \multicolumn{2}{|c|}{ Validity } & \multicolumn{2}{|c|}{ Reliability } \\
\hline & & Content validity & $\begin{array}{l}\text { Other validity } \\
\text { measures }\end{array}$ & $\begin{array}{l}\text { Internal Consistency } \\
\text { (Cronbach's alpha) }^{*}\end{array}$ & $\begin{array}{l}\text { Other reliability } \\
\text { measures }\end{array}$ \\
\hline PINCOM-Q & $\begin{array}{l}\text { Interprofessional climate, } \\
\text { organisational culture, } \\
\text { organisational aims, } \\
\text { professional power, } \\
\text { group leadership and } \\
\text { motivation. }\end{array}$ & $\begin{array}{l}\text { Questions formulated } \\
\text { by author, reference } \\
\text { to another } \\
\text { questionnaire and } \\
\text { theoretical model, } \\
\text { some colleagues } \\
\text { reviewed items }\end{array}$ & $\begin{array}{l}\text { Construct Validity - } \\
\text { PCA }\end{array}$ & $\begin{array}{l}\text { Total }=0.87 \\
\text { Sub scales }=0.84 \\
0.64,0.80,0.82,0.69 \\
0.88\end{array}$ & \\
\hline TACT Scale & $\begin{array}{l}\text { Exploitation, exploration, } \\
\text { conflict, constructive } \\
\text { controversy, } \\
\text { psychological safety, } \\
\text { goal agreement, } \\
\text { information accessibility, } \\
\text { encounter preparedness, } \\
\text { consumer centred care. }\end{array}$ & $\begin{array}{l}\text { Use of conceptual } \\
\text { model and interviews } \\
\text { of target population to } \\
\text { develop items, expert } \\
\text { panel reviewed. }\end{array}$ & $\begin{array}{l}\text { Construct Validity- } \\
\text { EFA, and CFA } \\
\text { Convergent validity } \\
\text { examined } \\
\text { Discriminant validity } \\
\text { examined } \\
\text { Predictive validity } \\
\text { (TACT scale and staff } \\
\text { outcomes) examined }\end{array}$ & $\begin{array}{l}\text { Total not provided. } \\
\text { Subscales from wave } \\
\text { three: } 0.91,0.75 \text {, } \\
0.79,0.76,0.85,0.80 \text {, } \\
0.76,0.84,0.70 \text {. }\end{array}$ & $\begin{array}{l}\text { Within team } \\
\text { agreement }\left(r_{w g}=0.51 \text {, }\right. \\
0.69,0.82,0.82,0.94 \text {, } \\
0.70,0.61,0.88,0.79) \text {. } \\
\text { Test-retest; the overall } \\
\text { goodness to fit was } \\
\text { acceptable }\end{array}$ \\
\hline IITC-ESMH & $\begin{array}{l}\text { Reflection on process, } \\
\text { professional flexibility, } \\
\text { newly created } \\
\text { professional activities, } \\
\text { and role independence. }\end{array}$ & $\begin{array}{l}\text { Refinement of The } \\
\text { Index of } \\
\text { Interdisciplinary } \\
\text { Collaboration } \\
\text { (Bronstein, 2002), } \\
\text { plus review of the } \\
\text { literature. }\end{array}$ & $\begin{array}{l}\text { Construct Validity - } \\
\text { EFA }\end{array}$ & $\begin{array}{l}\text { Total not provided. } \\
\text { Subscales }=0.91 \\
0.91,0.84,0.80\end{array}$ & \\
\hline TDMQ & $\begin{array}{l}\text { Decision making, team } \\
\text { support, learning, } \\
\text { developing quality } \\
\text { services. }\end{array}$ & $\begin{array}{l}\text { Review of literature } \\
\text { and focus groups, } \\
\text { then piloted with } \\
\text { experts. }\end{array}$ & $\begin{array}{l}\text { Construct Validity - } \\
\text { PCA }\end{array}$ & $\begin{array}{l}\text { Total }=0.96 \\
\text { Subscales }=0.90 \\
0.91,0.83,0.88\end{array}$ & $\begin{array}{l}\text { Inter correlation } \\
\text { coefficient for } \\
\text { subscales }=0.77,0.94 \text {, } \\
0.52,0.74 \text {. }\end{array}$ \\
\hline $\begin{array}{l}\text { PINCOM-Q } \\
(\text { revised })^{27} \\
\text { Rousseau et } \\
\text { al. }\end{array}$ & $\begin{array}{l}\text { Individual aspects: } \\
\text { Professional power, role } \\
\text { expectations, personality } \\
\text { style, and work } \\
\text { motivation. Group } \\
\text { Aspects: leadership, } \\
\text { coping abilities, } \\
\text { communication and } \\
\text { social support. } \\
\text { Organisational Aspects: } \\
\text { organisational aims, } \\
\text { environment, culture and } \\
\text { domain. }\end{array}$ & & & $\begin{array}{l}\text { Total = } 0.94 . \\
\text { Subscales: } \\
\text { Individual }=0.793 \text {, } \\
\text { Group = 0.907, } \\
\text { Organisational = } \\
0.864 \text {. }\end{array}$ & \\
\hline
\end{tabular}




\begin{tabular}{|c|c|c|c|c|c|}
\hline $\begin{array}{l}\text { PPE } \\
\text { (revised) } \\
\text { Scale }\end{array}$ & $\begin{array}{l}\text { Handling disagreement } \\
\text { and conflict, internal } \\
\text { work motivation, control } \\
\text { over practice, leadership } \\
\text { and autonomy in clinical } \\
\text { practice, staff } \\
\text { relationships with } \\
\text { physicians, teamwork, } \\
\text { cultural sensitivity, and } \\
\text { communication about } \\
\text { patients }\end{array}$ & $\begin{array}{l}\text { Original version - } \\
\text { theoretical model and } \\
\text { content validity panel }\end{array}$ & $\begin{array}{l}\text { Construct Validity - } \\
\text { PCA }\end{array}$ & $\begin{array}{l}\text { Total }=0.93 \\
\text { Sub scales= 0.88, } \\
0.86,0.82,0.83,0.79, \\
0.78,0.78,0.80 .\end{array}$ & \\
\hline CPAT & $\begin{array}{l}\text { Mission, meaningful } \\
\text { purpose, goals. General } \\
\text { relationships. Team } \\
\text { leadership. General role } \\
\text { responsibilities and } \\
\text { autonomy. } \\
\text { Communication and } \\
\text { information exchange. } \\
\text { Community linkages and } \\
\text { coordination of care. } \\
\text { Decision making and } \\
\text { conflict management. } \\
\text { Patient involvement. }\end{array}$ & $\begin{array}{l}\text { Review of the } \\
\text { literature, expert } \\
\text { opinion, pilot test of } \\
\text { sample examining } \\
\text { content through } \\
\text { additional questions. }\end{array}$ & $\begin{array}{l}\text { Construct Validity - } \\
\text { EFA, and CFA }\end{array}$ & $\begin{array}{l}\text { Total not provided } \\
\text { Sub scales }=0.88, \\
0.89,0.80,0.81,0.84 \text {, } \\
0.76,0.67,0.87\end{array}$ & \\
\hline MIIC & $\begin{array}{l}\text { Independence and } \\
\text { flexibility, newly created } \\
\text { activities, collective } \\
\text { ownership of goals, and } \\
\text { reflections on process. }\end{array}$ & $\begin{array}{l}\text { Development of Index } \\
\text { of Interdisciplinary } \\
\text { Collaboration } \\
\text { instrument, use of } \\
\text { conceptual } \\
\text { framework, and } \\
\text { reviewed by expert } \\
\text { panel. }\end{array}$ & & $\begin{array}{l}\text { Total }=0.935 \\
\text { Sub scales }=0.867 \\
0.767,0.795,0.791\end{array}$ & \\
\hline $\begin{array}{l}\text { Adapted ICU } \\
\text { nurse- } \\
\text { physician } \\
\text { (PACE } \\
\text { teams) }\end{array}$ & $\begin{array}{l}\text { Leadership, } \\
\text { communication, } \\
\text { coordination, conflict } \\
\text { management, team } \\
\text { cohesion, perceived } \\
\text { team effectiveness. }\end{array}$ & $\begin{array}{l}\text { Questions reviewed } \\
\text { by specialist in } \\
\text { education and English } \\
\text { as a second } \\
\text { language, and by an } \\
\text { expert panel. }\end{array}$ & $\begin{array}{l}\text { Predictive validity } \\
\text { examined }\end{array}$ & $\begin{array}{l}\text { Total not provided } \\
\text { Subscales= } 0.81, \\
0.82,0.76,0.76,0.82 \text {, } \\
0.89 .\end{array}$ & \\
\hline $\begin{array}{l}\text { Jones } \\
\text { Synergy } \\
\text { Scale }\end{array}$ & Synergy & $\begin{array}{l}\text { Focus groups use to } \\
\text { identify item themes, } \\
\text { content analysis with } \\
\text { inter coder reliability= } \\
85-100 \%\end{array}$ & $\begin{array}{l}\text { Construct Validity - } \\
\text { PCA } \\
\text { Convergent validity } \\
\text { examined } \\
\text { Discriminant validity } \\
\text { examined } \\
\text { Concurrent validity- } \\
\text { correlation with } \\
\text { similar scale = } 0.82 \text {, } \\
p=0.01\end{array}$ & Total $=0.91$. & \\
\hline $\begin{array}{l}\text { Jones Trust } \\
\text { Scale }\end{array}$ & $\begin{array}{l}\text { Positive trust and } \\
\text { mistrust. }\end{array}$ & $\begin{array}{l}\text { Focus groups use to } \\
\text { identify item themes, } \\
\text { content analysis with } \\
\text { inter coder reliability= } \\
85-100 \%\end{array}$ & $\begin{array}{l}\text { Construct Validity - } \\
\text { PCA }\end{array}$ & Total $=0.91$ & \\
\hline
\end{tabular}




\section{Results of the Critical Appraisal of the Studies}

Full agreement (100\%) between the reviewers was reached regarding the critical appraisal of studies. Critical appraisal findings varied across tools and studies (Table 3 ) for the six categories (purpose, validity, reliability, external validity, layout, and piloting) (Appendix 3). While the critical appraisal scores varied in terms of absolute numerical value, there was similar patterning with respect to distribution of percentages across items (Table 3). The average total score for the 11 articles was 43.1 out of a possible $70(62 \%)$, with total average scores ranging from $39.75(56 \%)$ to $54.25(78 \%)$.

Many of the articles and questionnaires lacked sufficient detail on psychometric properties with the lowest percentages seen in the validity (mean score 8.4/16, 53\%) and reliability (mean score 8.3/13,64\%) categories. All articles represented reasonable external validity, with an average of $77 \%$ (15.3/20). External validity was determined in relation to our setting of Children's Centres and may vary when determined for other settings. Ødegård (2006) and Rousseau et al (2012) both demonstrated the highest score on external validity of the PINCOM-Q including the desired constructs (related to adequate representation of health and other disciplines, team members' employed by more different agencies, and developed in a child-based setting).26-27 Ødegård's PINCOM-Q received the highest score overall from the critical appraisal process and was deemed most appropriate for children's services. 26

\begin{tabular}{|c|c|c|c|c|c|c|c|}
\hline \multicolumn{8}{|c|}{ Table 3 - Critical Appraisal Scores of the Included Studies } \\
\hline $\begin{array}{l}\text { Review criteria } \\
\text { (total possible score) }\end{array}$ & $\begin{array}{c}\text { Purpose } \\
\text { (4) }\end{array}$ & $\begin{array}{l}\text { Validity } \\
\text { (16) }\end{array}$ & $\begin{array}{l}\text { Reliability } \\
\text { (13) }\end{array}$ & \begin{tabular}{|c|} 
External \\
Validity (20)
\end{tabular} & $\begin{array}{l}\text { Layout } \\
\text { (5) }\end{array}$ & $\begin{array}{l}\text { Piloting } \\
\text { (12) }\end{array}$ & $\begin{array}{l}\text { Total } \\
(70)\end{array}$ \\
\hline $\begin{array}{l}\text { PINCOM-Q } \\
\text { (Ødegård, 2006) }\end{array}$ & 4 & 10.5 & 7 & 17.5 & 3.75 & 11.5 & 54.25 \\
\hline \begin{tabular}{|l|} 
TACT \\
(Wholey et al., 2012)
\end{tabular} & 4 & 14 & 5.5 & 10 & 2.75 & 10.25 & 46.5 \\
\hline $\begin{array}{l}\text { TDMQ } \\
\text { (Batorowicz \& Shepherd, } \\
\text { 2008) }\end{array}$ & 4 & 7.5 & 10 & 9.5 & 3 & 10.5 & 44.5 \\
\hline $\begin{array}{l}\text { IITC-ESMH } \\
\text { (Mellin et al., 2010) }\end{array}$ & 4 & 10 & 3 & 13.5 & 4.75 & 9 & 44.25 \\
\hline $\begin{array}{l}\text { Jones Synergy Scale } \\
\text { (Jones \& Barry, 2011a) }\end{array}$ & 3.5 & 11 & 5.25 & 9.5 & 4.25 & 10 & 43.5 \\
\hline \begin{tabular}{|l} 
PPE \\
(Erickson et al., 2004)
\end{tabular} & 4 & 9 & 6.5 & 9.5 & 5 & 8.5 & 42.5 \\
\hline $\begin{array}{l}\text { PINCOM-Q } \\
\text { (Rousseau et al., 2012) }\end{array}$ & 3 & 2 & 8 & 17.5 & 4 & 6.5 & 41 \\
\hline $\begin{array}{l}\text { CPAT } \\
\text { (Schroder et al., 2011) }\end{array}$ & 4 & 8.5 & 2.75 & 15 & 2.5 & 8 & 40.5 \\
\hline $\begin{array}{l}\text { Jones Trust Scale } \\
\text { (Jones \& Barry, 2011b) }\end{array}$ & 4 & 10 & 4.5 & 9.5 & 4.5 & 7 & 39.5 \\
\hline $\begin{array}{l}\text { MIIC } \\
\text { (Oliver et al., 2007) }\end{array}$ & 2 & 5.5 & 7.5 & 15 & 2.25 & 7 & 39.25 \\
\hline $\begin{array}{l}\text { Adapted ICU nurse-physician } \\
\text { (PACE teams) } \\
\text { (Temkin-Greener et al., 2004) }\end{array}$ & 4 & 4 & 4.75 & 13.25 & 4 & 8.75 & 38.75 \\
\hline Total average (percentage) & $3.7(93 \%)$ & $8.4(53 \%)$ & $8.3(64 \%)$ & $15.3(77 \%)$ & $3.7(74 \%)$ & $9.9(83 \%)$ & $43.1(62 \%)$ \\
\hline
\end{tabular}

\section{DISCUSSION:}

This is the first systematic review of tools available for interprofessional teams, comprised of health and other professionals, to self-evaluate their collaboration. In the past, most measures have had a narrow focus on teams consisting of a variety of health professionals only (e.g. the Observational Teamwork Assessment of Surgery). 19 Within the body of work published in the last 
decade, the findings of our review demonstrate that few psychometrically sound tools exist for more diverse professional groups working together.

All studies had considered issues relating to validity and reliability through the undertaking of psychometric testing; however, there was large variation in the quality of the measures and weak results seen for validity (average score in CAT 53\%) and reliability (average score in CAT 64\%). Validity and reliability sub categories received the poorest scores in the critical appraisal tool on average. The questionnaires received the best scores, on average, in the purpose (average score in CAT 93\%) and piloting (average score in CAT 83\%) sub categories of the critical appraisal tool. Therefore, each tool would benefit from further development to increase its validity and reliability, for example, more stringent psychometric testing, larger sample sizes, and application to different settings.

This review has identified that Ødegård's PINCOM-Q tool was the most appropriate tool, as reported and based on a critical analysis of the purpose and relevance of the tool and the psychometric properties.26 The PINCOM-Q was developed in Norway for child mental health settings, although it has been used in other settings.26,37 It is recommended that researchers or practitioners wanting to use this tool in their own settings should examine and appraise the relevance and psychometric properties of this tool prior to use.

This review provides an array of measures to assess teamwork in team environments that include professionals from different disciplines. This review can be of benefit to other researchers as it highlights some of the tools that already exist within the literature. These existing questionnaires can be used and/or adapted for a variety of teamwork settings. Firstly, these tools can be used for teams to reflect on their current teamwork practice in order to improve on and ensure effectiveness of their practice. 11 Secondly, being able to effectively measure collaboration will enable development of links between collaboration and effective practice, client outcomes, and team effectiveness, especially given the scarcity of documented outcomes for teams that comprise health and other professionals. Measuring collaboration effectively also provides the opportunity to assess changes over time and/or pre-post organisational structure changes, training, education, staff numbers and work place structures.

Consequently, the findings of this systematic review suggest that the recent increase in interest in teamwork and implementation of teamwork in practice, as well as its reported benefits, have not been reflected in the psychometric development of available tools documented in current literature, specifically in regards to health and other professionals working together in interprofessional teams.13

\section{Recommendations for Practice and Future Research}

It is recommended that all tools should undergo further analysis and development to be more robust. The PINCOM-Q appears to be the most worthy of further development and testing to ensure its suitability, reliability, and validity within the specific children service settings that contain interprofessional collaboration. Following further development, the tool can be used to facilitate team professional development and to strengthen their collaborative practice. Ten of the studies included in this review contained the questionnaire items or the tool was acquired upon direct contact with authors, which is encouraging for further development and use. In addition to improving the psychometric properties of these tools, various aspects can be considered for further development such as tool formats (e.g. electronic or paper-based), exploring the exact nature of collaboration (e.g. episodic collaboration versus long-term collaboration), the subscales of collaboration considered, and the type of professional groups involved. In addition, research into the relationship between the use of reflective, self-evaluation tools and client outcomes, as well as these tools and measuring actual collaboration, are areas that require further exploration.

\section{Limitations}

When considering the findings of this systematic review, there are a number of caveats to consider. Firstly, articles were included only if they were published in English within the last ten years, therefore excluding tools developed before this time. However, the exception to this was tools that were reviewed or modified within the last ten years from original and less recent versions. Subsequently, our findings are limited to, and should not be extrapolated beyond, these contexts. Secondly, although the search was extensive, there is a small possibility some publications were missed as a result of inconsistency in terms; however, reference lists were checked in order to guard against this. Finally, the critical appraisal tool used in this review was purposively developed by the research team using items from those successfully applied in other relevant studies. However, its psychometric properties have not been tested, and therefore, findings must be considered in this context.21-23 


\section{CONCLUSION:}

This paper identifies and appraises collaboration measurement tools available within the literature for teams consisting of health and other professionals in children's services and reports on their psychometric properties. The review suggests that despite the current need for increased interprofessional collaboration, especially in addressing complex health problems by incorporating health professionals and other team members, there is a lack of strong validation of existing measurement tools to assist teams in the development of their collaboration. In order to enable the use of rigorously developed tools for future use, there is a need for further studies assessing psychometric properties of these tools and adaptation of the tools for use in different interprofessional contexts. It is anticipated that practitioners and researchers will benefit from this review and its summary of available tools within the literature.

\section{References}

1. Zwarenstein M, Goldman J, Reeves S. Interprofessional collaboration: Effects of practice-based interventions on professional practice and healthcare outcomes. Cochrane Database of Syst Rev. 2009;3:1-33. [PMID: 19588316]

2. Braithwaite J, Westbrook M, Nugus P, Greenfield D, Travaglia J, Runciman W, Foxwell R, Boyce RA, Devinney T, Westbrook J. Four-year systems-wide intervention promoting interprofessional collaboration. BMC Health Serv Res. 2012;12:99. [PMID: 22520869]

3. Grumbach K, Bodenheimer T. Can health care teams improve primary care practice. JAMA. 2004;291(10):1246-51. [PMID: 15010447]

4. Lemieux-Charles L, McGuire WL. What do we know about health care team effectiveness? A review of the literature. Med Care Res and Rev. 2006;63(3):263-300. [PMID: 16651394]

5. Bower $P$, Campell $S$, Bojke $C$. Team structure, team climate and the quality of care in primary care: An observational study. Qual Saf Health Care. 2003;12:273-9. [PMID: 12897360]

6. Press F, Sumsion J, Wong S. Integrated Early Years Provision in Australia. 2010. Australia, NSW, Bathurst: Charles Sturt University, http://www.cscentral.org.au/Resources/Publications/FinalCSUreport.pdf. Accessed January 30, 2017.

7. Horwarth J, Morrison T. Collaboration, integration and change in children's services: Critical issues and key ingredient. Child Abuse Negl. 2007;31:55-69. [PMID: 17210177]

8. Nichols S, Jurvanssu S. Partnership in integrated early childhood services: an analysis of policy framings in education and human services. Contemporary Issues in Early Childhood. 2008:9:117-30.

9. Hillier S, Civetta L, Pridham L. A systematic review of collaborative models for health and education professionals working in school settings and implications for training. Educ Health. 2010;23:1-12. [PMID: 21290358]

10. D'Amour D, Ferrada-Videl, M, San Martin Rodriguez L, Beaulieu MD. The conceptual basis for interprofessional collaboration: Core concepts and theoretical frameworks. J Interprof Care. 2005;19(Suppl 1):116-31. [PMID: 16096150]

11. Boshoff $\mathrm{K}$, Stewart $\mathrm{H}$. Key principles for confronting the challenges of collaboration in educational settings. Aust Occup Ther J. 2013;60(2):144-7. [PMID: 23551008]

12. Havyer R, Wingo M, Comfere N, Nelson D, Halvorsen A, McDonald F, Reed D. Teamwork assessment in internal medicine: A systematic review of validity evidence and outcomes. J Gen Intern Med. 2014;29(6):894-910. [PMID: 24327309]

13. Valentine MA, Nembhard IM, Edmondson AC. Measuring teamwork in health care settings: A review of survey instruments. Med Care. 2013;53(4):16-30. [PMID: 24189550]

14. Mackay S. The role perception questionnaire (RPQ): A tool for assessing undergraduate students' perceptions of the role of other professions. J Interprof Care. 2004;18(3):289-302. [PMID: 15369972]

15. Reeves S, Perrier L, Goldman J, Freeth D, Zwarenstein M. Interprofessional education: effects on professional practice and healthcare outcomes (updated). Cochrane Database Syst Rev. 2013 Mar 28;(3):CD002213. [PMID: 23543515]

16. Thannhauser J, Russell-Mayhew S, Scott, C. Measures of interprofessional education and collaboration. J Interprof Care. 2010;24(4):336-49. [PMID: 20540613]

17. Frankel A, Gardner R, Maynard L, Kelly A. Using the Communication and Teamwork Skills (CATS) assessment to measure health care team performance. Jt Comm J Qual Patient Saf. 2007;33(3):549-58. [PMID: 17915529]

18. Healy AN, Undre S, Vincent CA. Developing observational measures of performance in surgical teams. Qual Saf Health Care. 2004;13(Suppl 1):i33-40. [PMID: 15465953]

19. Schroder C, Medves J, Paterson M, Byrnes V, Chapman C, O'Riordan A, Pichora D, Kelly C. Development and pilot testing of the collaborative practice assessment tool. J Interprof Care. 2011;25(3):189-95. [PMID: 21182434]

20. Crisp N, Chen L. Global Supply of Health Professionals. New Eng J Med. 2014;370:950-7. [PMID: 24897096]

21. Fox $A$, Reeves $S$. Interprofessional collaborative patient-centred care: a critical exploration of two related discourses. $J$ Interprof Care. 2015;29(2):113-8. [PMID: 25180630]

22. Moher D, Liberati A, Tetzlaff J, Altman DG, The PRISMA Group. Preferred reporting items for systematic reviews and metaanalyses: The PRISMA Statement. PLoS Med. 2009;6(7):1-6. [PMID: 19621072] 
23. Law M, Stewart D, Pollock N, Letts L, Bosch J, Westmorland M. Critical review form - Quantitative studies. [updated 1998; cited June 2014]. Available from: http://www.srs-mcmaster.ca/Portals/20/pdf/ebp/quanreview.pdf. Accessed January 30, 2017.

24. Boynton PM, Greenhalgh T. Hands-on guide to questionnaire research: Selecting, designing, and developing your questionnaire. BMJ. 2004;328:1312-5. [PMID: 15166072]

25. Stanley RM, Ridgers ND, Salmon J, Parish A, Okely A. Physical activity during recess: A systematic review. Am J Prev Med. 2012;43(3):320-8. [PMID: 22898126]

26. Harman-Smith Y, Brinkman S. Children's Centre evaluation. Interim Evaluation Report: Summary of Qualitative Evaluation Findings. Government report. Department for Education and Child Development. 2013. Adelaide, Australia.

27. West M, Boshoff K, Stewart H. A qualitative exploration of the characteristics and practices of interdisciplinary collaboration. SAJOT. 2016;46(2):37-43.

28. Ødegård A. Exploring perceptions of interprofessional collaboration in child mental health care. IJIC. 2006;6(4):1-13. [PMID: 17211492]

29. Rousseau C, Laurin-Lamothe A, Nadeau L, Deshaies S, Measham T. Measuring the quality of interprofessional collaboration in child mental health collaborative care. Int J Integr Care. 2012;12(1):1-8. [PMID: 22371692]

30. Jones J, Barry MM. Developing a scale to measure synergy in health promotion partnerships. Glob Health Promot. 2011a;18(2):36-44. [PMID: 21596938]

31. Jones J, Barry MM. Developing a scale to measure trust in health promotion partnerships. Health Promot Int. 2011b;26(4):484-91. [PMID: 21339194]

32. Mellin EA, Bronstein L, Anderson-Butcher D, Amorose AJ, Ball A, Green J. Measuring interprofessional team collaboration in expanded school mental health: Model refinement and scale development. J Interprof Care. 2010;24(5):514-23. [PMID: 20594069 ]

33. Wholey DR, Zhu X, Knoke D, Shah P, Zellmer-Bruhn M, Witheridge TF. The teamwork in assertive community treatment (TACT) scale: Development and validation. Psychiatr Serv. 2012;63(11):1108-17. [PMID: 22948786]

34. Oliver DP, Wittenberg-Lyles EM, Day M. Measuring interdisciplinary perceptions of collaboration on hospice teams. Am J Hosp \& Palliat Care. 2007;24(1):49-53. [PMID: 17347505]

35. Temkin-Greener H, Gross D, Kunitz SJ, Mukamel D. Measuring interdisciplinary team performance in a long-term care setting. Med Care. 2004;42(5):472-81. [PMID: 15083108 ]

36. Batorowicz B, Shepherd TA. Measuring the quality of transdisciplinary teams. J Interprof Care. 2008;22(6):612-20. [PMID: 19012141]

37. Erickson JI, Duffy ME, Gibbons MP, Fitzmaurice J, Ditomassi M, Jones D. Development and psychometric evaluation of the Professional Practice Environment (PPE) scale. J Nurs Scholars. 2004;36(3):279-85. [PMID: 15495499]

38. Strype J, Gundhus H, Egge M, Ødegård A. Perceptions of Interprofessional Collaboration. Professions \& Professionalism. 2014;4(3):808-20. 